\title{
QUASI-INVARIANT CONVERGENCE FOR DOUBLE SEQUENCE
}

\author{
AlAUDDIN DAFADAR* AND D. K. GANGULY
}

Abstract. In this paper we introduce the concept of quasi-invariant convergence and quasiinvariant statistical convergence of double sequence in a normed space and we shall present a characterization of a bounded sequence to be quasi-invariant convergent.

Mathematics subject classification (2010): 40B05, 40A05.

Keywords and phrases: Invariant mean, Banach limit, invariant limit, quasi invariant convergent, quasi invariant statistical convergent.

\section{REFERENCES}

[1] Biswa Ranjan Datta and Absos Ali Shaikh, Generalized almost statistical convergence, Real Anal. Exchange, 45, 2 (2020), 439-452.

[2] C. Caken, B. Altay, M. Mursaleen, The $\sigma$-convergent and $\sigma$-core of double sequences, Applied Mathematics Letter, 19, (2006), 1122-1128.

[3] D. K. Ganguly and Alauddin Dafadar, On quasi statistical convergence of double sequence, Gen. Math. Notes, 32, 2 (February 2006), 42-53.

[4] D. K. Ganguly, B. Biswas and Alauddin Dafadar, Some properties of a function connecting to exponent of convergence for double sequences, Journal of Classical Analysis, 10, 2 (November 2017), 109-117.

[5] D. K. GANGUly AND A. DAFADAR, On strong matrix summable double sequence space with respect to modulus and statistical convergence, J. Cal. Math. Soc., 12, 1 (2016), 9-20.

[6] E. Savas, Some Sequence Space and Statistical Convergence, Int. J. Math. and Math. Sci., 29, 5 (2002), 303-306.

[7] E. SAVAS, F. NuRry, On $\sigma$-statistical convergence and lacunary $\sigma$-statistical convergence, Math. Slovaca, 43, 3 (1993), 309-315.

[8] E. Savas, Some sequence space involving invariant means, Indian J. Pure Appl. Math., 31, (1989), $1-8$.

[9] E. SAVAS, Quasi-invariant convergent in normed space, Annals of the University of Cralova, Mathematics and computer science series, 41, 1 (2014), 1-5.

[10] F. NURRY, E. S AVAS, Invariant statistical convergence ana A-invariant statistical convergence, Indian J. Pure Appl. Math., 10, (1994), 267-294.

[11] H. FAST, Sur la convergence, Colloq. Math., 2, (1951), 241-244.

[12] I. J. Schoenberg, The integrability of certain functions and related summability methods, Amer. Math. Monthly, 66, (1959), 361-375.

[13] M. BASARIR (ELAZIG), On the strong almost convergence of double sequence, Periodica Mathematica Hungarica, 30, 2 (1995), 99-105.

[14] M. Mursaleen AND O. H. H. Edely, Almost convergence and a core theorem for double sequences, J. Math. Anal. Appl., 293, (2004), 532-540.

[15] M. Mursaleen and Osama H. H. Edely, On the invariant mean and statistical convergence, Appl. Math. Letters, 22, (2009), 1700-1704.

[16] M. MURS ALEEN et.al., Generalized statistical convergence and statistical core of double sequences, Acta Mathematica Sinica, 26, (November 2010), 2131-2144.

[17] N. Subramanian AND AYHAn Esi, On triple sequence of Bernstine operator of weighted rough $I_{\lambda}$-convergence, Journal of Classical Analysis, 13, 1 (November 2018), 45-62. 
[18] R. A. RAIMI, Invariant means and invariant matrix method of summability, Duke Math. J., 30, (1963), $81-94$.

[19] T. SALAT, On statistically convergent sequences of real numbers, Math. Slovaca, 30, (1980), 139-150. 